\title{
La interacción con los muertos en la cultura Chancay
}

\author{
Łukasz Majchrzak, Pieter Dennis van Dalen Luna
}

\begin{abstract}
Resumen
Presentamos los resultados del estudio estratigráfico y forense de los fardos y cuerpos pertenecientes a la cultura Chancay, excavados en los últimos años en el valle de Huaura. Nos enfocamos en la identifi cación de los indicadores del contacto directo con el muerto y con su fardo. Los documentos coloniales informan de que existía tal práctica, y que, al menos en la sierra, los cuerpos de los antepasados participaban en fie-stas y otras ceremonias religiosas. Nuestros resultados indican que algunos de los fardos presentan eviden-cias de un contacto póstumo, para depositar nuevas capas de textiles. Los cuerpos ubicados en los fardos estaban articulados, lo que permite excluir que se sacaba los cuerpos mismos. Sin embargo, identificamos algunos casos en que se registró la ausencia del cráneo. Este fenómeno pudo ser la consecuencia del uso ritual de las cabezas momificadas. Para un mejor entendimiento de todos estos datos, aprovechamos con una introducción teorética, en base de que proponemos algunas interpretaciones de nuestros resultados.
\end{abstract}

\begin{abstract}
INTERACTION WITH THE DEAD IN CHANCAY CULTURE

We present the results of the stratigraphic and forensic study of funerary bundles and human remains of the Chancay culture, excavated in recent years in the Huaura valley. We focus on the identification of the indicators of direct contact with the dead and his bundle. he colonial documents inform about that such practice existed, and, at least in the highlands, bodies of the ancestors participated in fiestas and other religious ceremonies. Our results show that some of the bundles present evidence of the posthumous inte-raction to add new textiles. However, the bodies placed inside the bundles were fully articulated, which excludes the possibility that they were being removed. Nevertheless, we identified some cases where the skull was absent. his phenomenon could be the consequence of the ritual use of mummified heads. For a better understanding of this data, we present a theoretic introduction, on the basis that we propose some interpretation of our results.
\end{abstract}

Łukasz Majchrzak - Instituto de Arqueología, Universidad Jaguellona, archeoluk@gmail.com, Majchrzak https://orcid.org/0000-0001-9168-1427

Pieter Dennis van Dalen Luna - Director del Proyecto Arqueológico Cerro Colorado Los Pinos. Docente Asociado a Dedicación Exclusiva del Departamento Académico de Arqueología,

Universidad Nacional Mayor de San Marcos. Past Vice Decano Nacional del Colegio Profesional de Arqueólogos del Perú. E mail: pvandalen2@hotmail.com, van dalen https://

orcid.org/0000-0002-2498-9242 


\section{Introducción}

En la década de 1980 el proceso de convulsión sociopolítica por la que atravesaba el Perú, hizo que la intensidad de las investigaciones arqueológicas disminuyó significativamente, situación que se mantuvo hasta la década del 90. Si antes los estudios sobre la cultura Chancay eran mínimas, en estas dos décadas casi desaparecieron; casi, pues gracias a la figura del arqueólogo polaco Andrzej Krzanowski, con sus investigaciones en los valles de Chancay y Huaura, volvieron a poner en el debate académico la importancia de esta cultura, sus asentamientos y manifestaciones culturales. La publicación el año 1991 del libro "Estudios sobre la cultura Chancay, Perú", marcó un hito importante para el conocimiento de esta entidad sociopolítica andina, y a la vez creó conciencia entre investigadores, estudiantes y autoridades para la protección de sus sitios arqueológicos. Por ello consideramos que Andrzej Krzanowski, junto a Julio C. Tello y Hans Horkheimer, son los que más han aportado en el conocimiento de esta sociedad Chancay.

La interacción con el muerto y la manipulación póstuma de sus restos constituían una parte importante de religión en las sociedades agrícolas prehistóricas tanto en Europa y Asia (Goring-Morris 2002; Chapman y Gaydarska 2006; Hollund et al. 2018), como en Mesoamérica (Medina y Vargas 2007; Fitzsimmons 2009). Los cuerpos de ancestros eran vistos como semillas y la fuente del poder fecundador. En consecuencia, el uso de sus huesos en ceremonias diferentes, incluyendo rituales agrícolas era común (Eliade 1974: 129 - 131; Szyjewski 2016: 430 - 434). En los Andes Centrales, los documentos coloniales no dejan ninguna duda en cuanto a la importancia de los restos físicos de ancestros en el mundo andino prehispánico. En su crónica, el extirpador de idolatría Pablo Joseph de Arriaga, con respeto a la provincia de Cajatambo (en sus límites se ubicaban los valles de Huaura y Chancay) dice:

"Despues de estas huacas de piedra la mayor veneracion y adoracion es la de sus malquis, que en los llanos llaman munaos, que son los huesos o cuerpos enteros de sus progenitores gentiles, que ellos dicen que son hijos de las huacas, los cuales tienen en los campos en lugares muy apartados, en los machays, que son sus sepulturas antiguas, y algunas veces los tienen adornados con camisetas muy costosas o de plumas de diversos colores o de cumbi. Tienen estos malquis sus particulares sacerdotes y ministros y les ofrecen los mismos sacrificios y hacen las mismas fiestas que a las huacas" (Arriaga 1968 [1621]: 203).

Como en todas las sociedades preindustriales entonces, el culto a los ancestros ocupaba un lugar fundamental en las religiones andinas precolombinas. Las crónicas y estudios etnohistóricos informan de que la visión de la composición anímica de los Andinos tuvo un carácter dividual, con un ser humano compuesto tanto del cuerpo, como de varias sustancias y calidades invisibles (cf. Fowler 2004). El componente más importante era el "camac", la energía vital cual animaba a 
todas las cosas en el universo, incluyendo los humanos. Como informa Taylor, algunas personas poseían más camac que otros; esas personas - a quienes se llamaba camasca - podían manipular a esta energía (Taylor 2000). Como se puede deducir de los relatos coloniales, los muertos fueron percibidos como camasca, poseyendo una capacidad de transmitir camac y fertilizar a los humanos, animales y la tierra (Altamirano y Arguedas 2016). El mismo término "malqui" que se daba a los ancestros significaba "planta tierna para crecer", como indica el diccionario de Gonzalo Holquin (2007 [1608]: 41). Díaz (2016) llama atención a las similitudes entre el ciclo vital de los humanos y las plantas en el pensamiento andino; tras la muerte uno queda como una semilla: seco y muerto, pero con la capacidad de brotar vida nueva. El poder fertilizante de los antepasados los posicionaba como un elemento esencial y necesario en el universo andino, donde el hombre tuvo que hacer frente a las condiciones medioambientales extremas. Los estudios modernos aseguran también, que el muerto tiene una vinculación con la lluvia. Plasencia (2015) cuenta de su visita en el cementerio de Huayllay Grande (Huancavelica), donde los campesinos sacaron de su tumba un cuerpo de una mujer fallecida, para que se parase la lluvia por el periodo de la fiesta patronal. La creencia de que los restos humanos tienen un enlace directo con los fenómenos meteorológicos posiblemente tiene raíces en los tiempos prehispánicos.

\section{Las costumbres funerarias en los Andes a fines de la época prehispánica}

Cuando llegaron los españoles a los Andes Centrales, existían dos maneras diferentes de enterramiento con respeto a la sierra y a la costa. En la sierra se metía los cuerpos en unos cueros animales o envolvía con mantas, poniéndolos después en las chulpas. Otro patrón era en la costa, donde se colocaba los cuerpos envueltos con mantas en los pozos excavados en los "arenales", ósea, en el desierto, tapándolos con arena (Cieza 1964 [1552]:351; Poma 1980 [1615]: 290 - 299). Como mencionamos antes, algunos cronistas, como el citado Arriaga, informan de los diferentes términos dados a los cuerpos de antepasados: malqui en la sierra, y munao en la costa. La segunda palabra posiblemente proviene del idioma quingnam que se hablaba en la costa norte y parte del valle de Huaura y significa "abuelo" o "hermano", informa Salas (Salas 2008; véase también Makowski 2010). Lamentablemente no hay muchas informaciones sobre los "munao" en los documentos coloniales; ¿fue una percepción diferente de su composición anímica, su rol en la sociedad, o solo se diferenciaban por el nombre de los "malqui" de la sierra?

En la sierra, como narra e ilustra Poma, fue una costumbre común sacar a los cuerpos de muertos para que tomasen parte en las fiestas o batallas, abasteciéndolos de nueva ropa y comida. Igualmente, los documentos escritos por "extirpadores de idolatría" no dejan dudas de la importancia del contacto físico con los "malqui". Un modo interesante de interactuar con el muerto fue cortar su rostro para convertirlo 
en una máscara llamada huayo, una costumbre, según Berraza (2009), mucho más antigua que los fines del siglo XVI, cuando se la menciona en el famoso manuscrito Haurochiri (Ávila 1962 [1584]). Las excavaciones de van Dalen en la sierra de Huaral confirman que esas costumbres fueron practicadas también entre los vecinos serranos de los Chancay, los Atavillos (van Dalen 2019).

En la costa tenemos que ver con una situación mucho menos clara. Mientras que varios documentos coloniales informan cómo se realizaba el entierro, la información sobre una interacción póstuma con los antepasados es muy escasa. Un relato valioso podemos encontrar en la crónica de Bartolomé de las Casas (1892 [1552]: 113):

"Encima de las sepolturas edificaban ciertas paredes y casas sin cubierta del mismo tamaño, y allí echaban la comida ordinaria y quemaban ovejas y sebo y conejos y otras cosas, como por sufragios que, según creían se consolaban las ánimas."

Como sabemos, el obispo de Chiapas nunca había estado en los Andes Centrales, y esta descripción probablemente le fue entregada por su amigo, fray Domingo de Santo Tomás, quien pasó más de 30 años en el Virreinato del Perú, en especial en los pueblos del valle Chancay como Santo Domingo de Real Aucallama (antiguo territorio de la cultura Chancay). Krzanowski (2008: 110 - 116) identificó en los cementerios prehispánicos ubicados en este valle las estructuras que corresponden a la descripción del cronista. También las excavaciones conducidas desde el año 2009 por van Dalen en el cementerio de Macatón indican la presencia de la arquitectura ceremonial, posiblemente construida para venerar a los ancestros, tratándose de amplios recintos con muros de tapiales que están parcelando las áreas ceremoniales y funerarias. Isbell (1997: 113) informa entonces que, en contraste a la sierra, en la costa el culto a los ancestros no incluía el contacto directo con sus cuerpos. Sin embargo, no se puede olvidar de que en la costa pues existía una larga y antigua tradición de la manipulación de cabezas humanas, como las de la cultura Nasca. Aunque llamados "cabezas trofeos", los cráneos humanos momificados, presentes tanto en la iconografía como en los contextos arqueológicos a partir de finales del periodo Formativo parecen tener una vinculación fuerte con la agricultura y la fertilidad de tierra, posiblemente también la lluvia, compartiendo entonces los rasgos de los malqui (Proulx 1999).

\section{Los fardos de la cultura Chancay}

La cultura Chancay se desarrolló en la costa norcentral peruana desde finales del Horizonte Medio (aprox. 900 AD), en el Periodo Intermedio Tardío (1000 - 1470 AD), Horizonte Tardío (1470 - 1533) y hasta los años sesenta del siglo dieciséis. A pesar que es muy conocida por sus extraordinarios textiles y llamativa 
cerámica del estilo Negro sobre Blanco, recién fue estudiada científicamente solo por algunos arqueólogos, como Julio C. Tello (2015), Hans Horkheimer (1970), Rosa Fung (1978), Peter Kaulicke (1997), Andrzej Krzanowski (1991; 2008) Pieter van Dalen (2008, 2012, 2016, 2017) y Alberto Bueno (2012). Krzanowski propuso una visión completa de esta cultura, abarcando problemas como patrones del asentamiento, rituales funerarios y su economía, finalmente dedicándose al estudio de los sitios amurallados en los valles de Huaura y Chancay (2016).

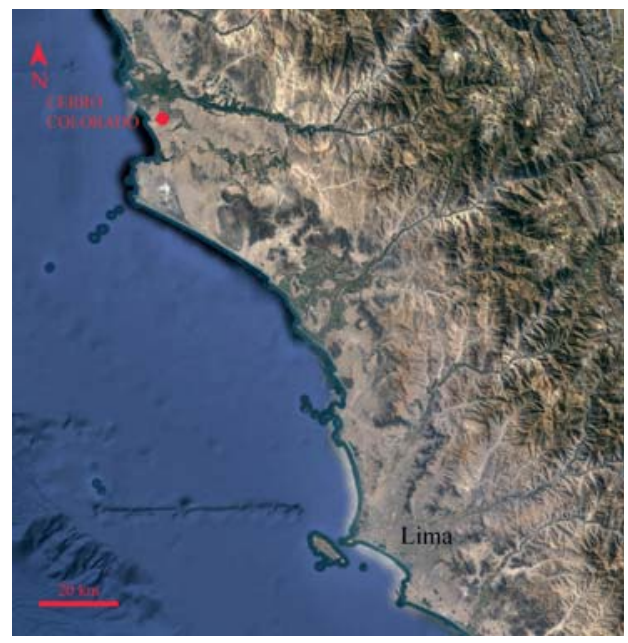

Fig. 1

A partir de los primeros años del

nuevo milenio, el equipo dirigido por Pieter van Dalen Luna ha excavado varios sitios arqueológicos (Cuyo, Lumbra, Macaton, Sacachispa, Andoma, Castillo de Pasamayo, Pampa de Animas, Walmay, Cerro Colorado, entre otros) pertenecientes al Periodo Intermedio Tardío y Horizonte Tardío en los valles de Chancay y Huaura (pertenecientes a la zona tradicionalmente llamada Norte Chico), lo que permitió definir varios rasgos de esta cultura. El proyecto arqueológico en el sitio de Cerro Colorado ubicado en el valle de Huaura (Fig. 1), por primera vez posibilitó excavar y registrar con metodología moderna los contextos funerarios intactos de la cultura Chancay, identificando numerosos contextos funerarios que se encontraban entre 1 y 2 metros de profundidad, con estructuras funerarias elaboradas sobre la capa arenosa o salitrosa. Estas estructuras funerarias contenían en su interior a los individuos, en su mayoría colocados al interior de fardos o paquetes funerarios, asociados a numerosos objetos arqueológicos que forman parte de las ofrendas colocadas durante el ritual funerario o en épocas posteriores como parte del culto a los ancestros. Muchos de estos materiales formaban parte de los implementos personales, laborales o sociales d elos individuos. El proyecto de van Dalen permitió también conocer las características de los fardos funerarios de la cultura Chancay, conformados por numerosas capas superpuestas de textiles y productos orgánicos, colocados con el objeto de "solidificar" al muerto, convirtiéndolo en una estructura compleja que abarca el cuerpo del individuo y las ofrendas depositarias por la sociedad en que vivió. Por lo general, el fardo funerario andino tenía la forma de un cilindro aplanado con extremos redondeados (van Dalen y Majchrzak 2019). Los principales constituyentes de cada fardo son los textiles, y su número puede ser más de treinta, cada uno constituyendo una capa. A veces, había una capa de algodón colocada entre ellas, probablemente para dar la forma deseada 
al fardo, así como muchos artefactos. El peso de los fardos de Cerro Colorado por lo general tiene entre 5 y 30 kilos, aunque algunos de ellos excedían los 50 . La longitud variaba de 30 a $40 \mathrm{~cm}$ en el caso de los niños, de 100 a $130 \mathrm{~cm}$ en el caso de los adultos, con un ancho de 20 a $70 \mathrm{~cm}$, y un espesor de 20 a $60 \mathrm{~cm}$. Los individuos ubicados en los fardos se encontraban en la posición de cúbito dorsal, con las extremidades inferiores flexionadas en rodillas, y las manos colocadas sobre el pecho o sobre el rostro (van Dalen et al. 2018; van Dalen y Majchrzak 2019). El rostro del individuo se cubría con láminas de cobre, y a menudo plata, y se llenaba la boca con une pieza de algodón mixto con pequeños fragmentos metálicos. Luego el cráneo se envolvía con gasas, y todo el cuerpo con textiles y capas de algodón. Más de $95 \%$ de todos los textiles ubicados en los fardos se ejecutaba a base de algodón, solo algunos fueron hechos de lana. La mayoría de los textiles fue llana $1 / 1$, aunque se notaba también la presencia de los tapices, ejecutados de algodón (urdimbres) y lana (tramas), decorados con motivos geométricos y zoomórficos (van Dalen y Majchrzak 2019). No se usaba palos ni cuerdas para soportar el peso del fardo, observados en los fardos de Ancón (Kaulicke 1997) y el valle de Chancay (van Dalen 2005); al parecer, la rigidez del paquete se obtenía apretando los mismos textiles alrededor del cuerpo. Entre los textiles se colocaban herramientas de madera para hilar: husos (ambos sexos, incluyendo los subadultos), y de vez en cuando, espadas para tejer (solo mujeres adultas). En cuanto a la condición biológica, se trata de una población enteramente dedicada al trabajo físico pesado, indicado por osteoartritis severa en las articulaciones (especialmente de la columna vertebral, codos y rodillas). Se identificó 70 hombres con exostosis (tumores óseos) en conductos auditivos externos, un indicador del trabajo en el mar, como pesca o recolección de moluscos (Silva - Pinto et al. 2013). Sin embargo, ninguno de esos individuos no poseía en su ajar funerario herramientas (como anzuelos, redes, flotadores) que podrían indicar tal trabajo.

Se realizaron análisis de datación radiocarbónica de los 32 fardos, lo que permitió definir la cronología del sitio, con el fechado más temprano de $1115 \pm 30$ BP (2б: 893 - 1025 d.C.) para el CF 38 (Unidad 42, Subunidad 6) y el más tardío de $495 \pm 30$ BP (2ఠ: 1411 - 1479 d.C.) para el CF 1 (Unidad 30, Subunidad 5). Los fardos más elaborados se ejecutaban a partir de la mitad del siglo XIII (Majchrzak et al., en prensa).

Se realizó también el estudio de los modos de momificación, para verificar si la sociedad Chancay practicaba la momificación intencional. Normalmente los intestinos se descomponen en las primeras semanas después de muerte, mientras otras partes como los huesos, la piel y el cabello pueden permanecer años o aun siglos (Pinheiro 2006). Para conocer la degradación de la piel de los individuos (e identificar maneras de momificación) se ha conducido estudio arqueométrico con la aplicación de FTIR - RS, SEM - EDX y GC - HPLC, las técnicas especializadas de física y química analítica, que permitieron identificar sustancias 
aplicadas a los cuerpos de muertos: yerbas, minerales de origen serrano, grasa animal, posiblemente también resinas. Los $5 \%$ de todos los individuos excavados por van Dalen tuvieron su rostro pintado con el cinabrio (Van Dalen et al. 2020). Esos bálsamos si pararon la descomposición de la piel, pero no se sabe si fueron aplicados con este fin. Excluimos la posibilidad de extracción de órganos, como no encontramos ningunos cortes en el abdomen o el rectum. Además, en algunos casos, ubicamos órganos momificados. Los cuerpos estudiados por nosotros seguían un modo de descomposición típico para los restos enterrados en el desierto, con la momificación parcial o entera de las extremidades y la parte anterior del tronco, y descomposición completa de su parte posterior (cf. Janaway et al. 2009). En cuanto a los ligamentos, se conservaban los de la rodilla y del codo, mientras los de la articulación del hombro y cadera normalmente estaban descompuestos, tanto como los del esqueleto axial.

\section{Materiales y métodos}

Durante el proyecto se recuperó más de 1700 contextos funerarios y estudiamos más de 500 fardos funerarios intactos, conociendo tanto su estratigrafía, como la condición biológica de la sociedad prehispánica de la zona de Huacho. El análisis estratigráfico de los fardos fue conducido según los procedimiento publicado por Frame et al. (2004). La determinación del sexo y edad de los individuos fue conducida según la metodología propuesta por Buikstra y Ubelaker (1994) para los adultos, y Cunningham et al. (2016) para los subadultos. La determinación e interpretación de cambios paleopatológicos fue conducida según los procedimientos descritos por Ortner (2003) y recién revisados por Buikstra (2019). Para verificar si el cuerpo fue sometido a la manipulación póstuma, se empleaba la tanatología desarrollada por Henry Duday (2009). Este método incluye la observación in situ de articulaciones del cuerpo para determinar dislocaciones posibles de huesos, algo que puede indicar que los restos de cadáver fueron movidos o disturbados después de su sepultura. Este método permite también entender las etapas y maneras de descomposición del cuerpo, lo que aumenta nuestro conocimiento del ritual funerario. El presente estudio se basa, entonces, en la observación de los cambios tafonómico en los fardos y cuerpos humanos, que podrían indicar un tipo de manipulación mencionada en las crónicas: los momentos cuando se cambiaba o añadía textiles, y cuando se sacaba o movía el cuerpo. El fardo funerario presenta una estratigrafía como todo contexto arqueológico, con la excepción de que mientras en el campo ubicamos una estratigrafía fundada sobre la roca madre como la más inferior y natural, en los fardos el primer nivel o la primera capa siempre está constituida por el cuerpo o esqueleto. En la estratigrafía tradicional, cualquier intervención posterior a la creación de una capa incluye su exposición y la destrucción parcial o completa. Eso se aplica parcialmente al fardo, con la condición que la deposición 
posterior de capas siguientes (como textiles) no tiene que causar el daño a las ya existentes. En otras palabras, para envolver con textiles nuevos un fardo enterrado un tiempo antes, se le podría sacar sin destruir los que ya envolvían al fardo. Sin embargo, no se puede excluir que con esta oportunidad se quitaba algunos textiles viejos, un acto que - si no estaban en el contacto directo con el cuerpo - pudo pasar sin dejar cualquier evidencia. En teoría, un fardo sometido a varios episodios de envoltura debería presentar textiles o artefactos ejecutados en estilos típicos para su época. Los 32 fechados radiocarbónicos ejecutados para Cerro Colorado abarcan un periodo entre los siglos X y XV a.C. Gracias a eso se pudo identificar que la mayoría de los tipos tecnológicos de textiles, así como los estilos decorativos, siguen a través de, por lo menos, tres siglos sin cambios mayores. Un método alternativo es la datación radiocarbónica de cada capa del fardo. Es un método costoso, y como indica el estudio recién publicado por Takigami et al. (2014), no permite resolver este problema por las limitaciones de la curva de calibración SHCal. La calibración de los fechados con la probabilidad de $95 \%$ normalmente da un intervalo de 120 - 150 años y todas las capas que constituyen un fardo pueden rendir los fechados que se superponen. No obstante, el estudio estratigráfico de los fardos - en algunos casos - permite identificar los momentos de la interacción.

Para verificar la manipulación del cuerpo del muerto, se observa la posición de sus partes, con el entendimiento de que varios huesos incluyendo el cráneo pueden cambiar su lugar por procesos tafonómicos naturales, también por el carácter del ambiente; por el cuerpo que se va a descomponer de manera diferente a la intemperie que siendo completamente cubierto por la tierra (Duday 2009). Los individuos de Cerro Colorado presentaban varios grados de momificación (van Dalen al. 2020). Sin embargo, el proceso de la descomposición normalmente afectaba articulaciones del esqueleto axial (incluyendo la articulación atlantooccipital), y - en la mayoría de los casos - también los del hombro y la cadera. Cualquiera interacción póstuma con el cuerpo - apertura del fardo, su transporte, participación en procesiones descritas por cronistas del siglo XVII - resultaría en el desplazamiento de los elementos, de los cuales articulaciones se habían descompuesto: vertebras, costillas, cabeza y las extremidades momificadas que cambiarían su posición con respeto al tronco.

\section{Resultados - Identificación de las prácticas póstumas en los fardos de la cultura Chancay}

El estudio estratigráfico de los fardos funerarios de Cerro Colorado, permitió identificar la costumbre de la deposición de textiles en la cima de algunos de ellos; a veces la calidad de esos materiales excedía los usados para construir el fardo mismo. No se trata de un patrón común, se registró tal situación en pocos casos no más. En este trabajo presentamos dos casos de este tipo: los CF 20 y "Gran Fardo" (Tabla 1). Sobre la cima del fardo descompuesto del hombre adulto mayor 
denominado por nosotros como "Gran Fardo" (Fig. 2), se ubicaban 37 telas (algunas decoradas) en un regular estado de conservación, y lo que separaba a la superficie del fardo y el primero de esos textiles fue una capa fina de arena, que por sus rasgos puede ser identificada como el suelo del sitio. ¿Fueron todos esos estratos - el fardo, la capa de tierra y los textiles - depositados en el mismo momento y en la misma ceremonia del enterramiento? ¿O eran al menos dos episodios diferentes, y la capa de arena sobre el fardo indica que eso tuvo que estar excavado para colocar los textiles? El fechado radiocarbónico de uno de los textiles colocados sobre la cima del fardo rindió datación de $654 \pm 30 \mathrm{BP}(2 \sigma$ : 1290 - 1395 d.C. [Majchrzak et al., en prensa]), pero este fechado indica el momento de la cosecha de algodón que se usó para tejer el textil. En el futuro ejecutaremos el siguiente fechado del fardo mismo, pero hay que recordar
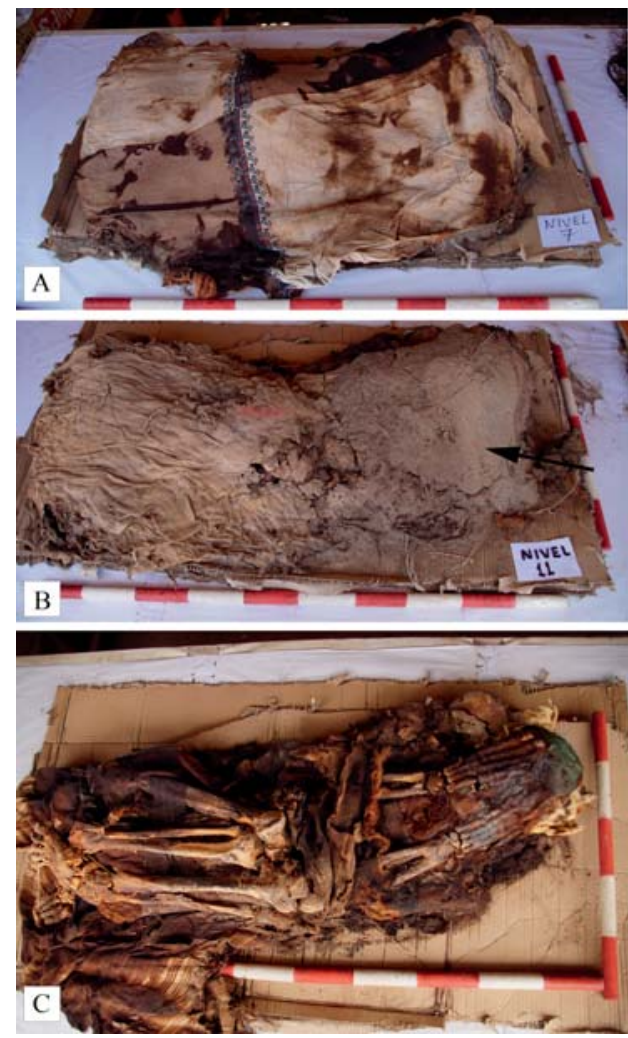

Fig. 2 los problemas con la calibración mencionados por Takigami y colegas (2014) - el peligro de que los fechados se sobreponen, especialmente si entre la deposición del fardo y la fabricación del textil no pasaron más que cien años. La presencia de una capa abundante de arena fue registrada también en el fardo del CF 20, Unidad 2 (Fig. 3). En este caso, el cuerpo estaba envuelto en cinco telas, con la capa de la piel con pelo de color ocre-amarillento (posiblemente de perro) metida entre dos de ellas. Sobre la tela exterior (la parte anterior del fardo) se ubicaba una capa de arena del mismo tipo que en el caso anterior, ósea, del suelo del sitio. Sobre esta capa se ubicaba un textil llano balanceado de color de naranja, con piezas de cobre cosidas a su superficie, y más arriba tres paños con decoración geométrica. Las dos últimas telas envolvían todo el fardo, con una red colocada sobre la superficie del textil exterior. El fechado (algodón del interior del fardo) rindió la datación de $800 \pm 30$ BP (2б: 1208 - 1280 d.C. [Majchrzak et al., en prensa]), no se ejecutó otro fechado para una de las capas exteriores. Sin embargo, la misma estratigrafía sugiere una de dos posibilidades: la deposición intencional de la capa de tierra durante la ceremonia funeraria o la excavación posterior al enterramiento, para colocar nuevos textiles. 

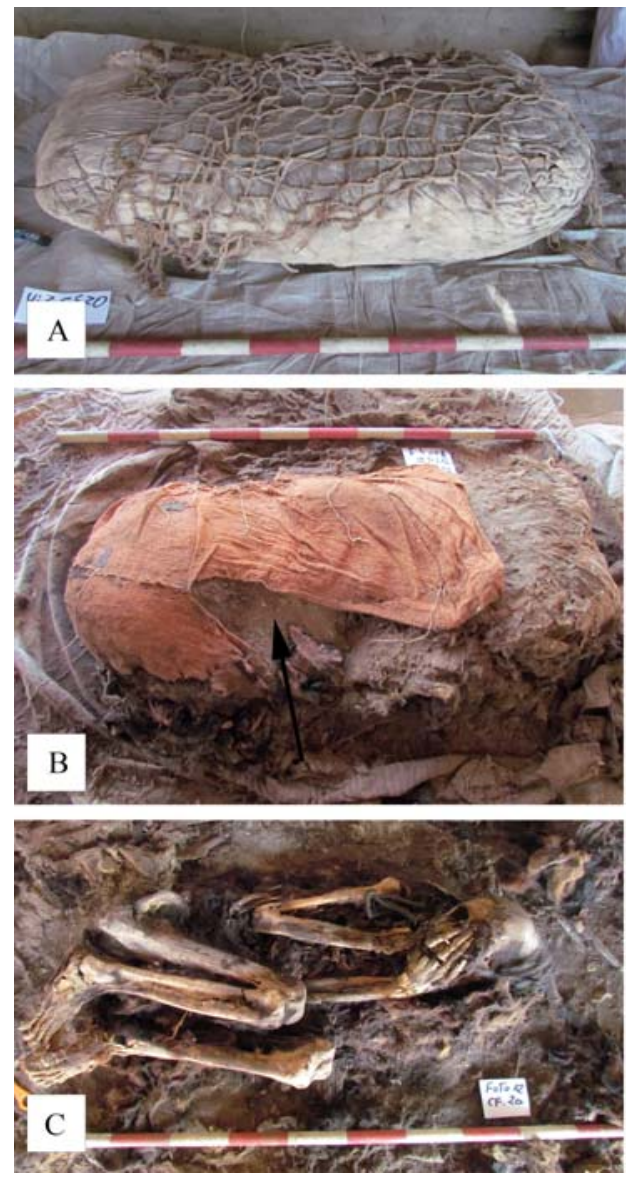

Fig. 3

Los individuos en los fardos cubiertos por capas adicionales de textiles presentaban la condición biológica común para todo el sitio, poseyendo numerosos marcadores de estrés físico típicos para la gente dedicada a un trabajo físico pesado, mayormente la pesca y navegación (hombres) y la actividad de tejer (mujeres). Sin embargo, en sus fardos se identificaba materiales de calidad muy excepcional, incluyendo adornos metálicos; algo lo que nos hace pensar que posiblemente tenemos que ver con una élite de artesanos y pescadores. ¿Fueron, entonces, esos textiles depositados sobre la cima del fardo en el momento de enterramiento, o un tiempo después? Como observamos, el estado de conservación de estos textiles contrastaba con los del fardo. Los fardos más antiguos seguían un modo de descomposición donde sus textiles se descomponían y pegaban unos con otros, creando una compactación con la superficie muy agrietada. Los textiles colocados sobre esos fardos, aun los que quedaban en el contacto directo con la superficie del fardo, no presentan tal grado de degradación como los que componían el fardo. Eso sugiere que esos textiles no estaban expuestos a los mismos factores que los fardos, posiblemente por su colocación posterior al enterramiento. Para sumar, entonces, en algunos casos aunque pocos - en Cerro Colorado, el cementerio de la cultura Chancay, tuvimos que ver con una situación estratigráfica que sugiere la posibilidad de una interacción con el fardo en un tiempo posterior al enterramiento.

Ahora nos cabe preguntar: ¿tuvimos que ver en el material excavado en Cerro Colorado con la manipulación de los restos humanos? Tanto en el caso de los dos contextos discutidos anteriormente, como en todos los demás fardos intactos, los individuos presentaban orden anatómico completo. De acuerdo con lo que presenta Duday (2009: 25 - 30), en Cerro Colorado los ligamentos de las articulaciones coxo-femoral, del hombro y las que conectan el cráneo al tramo cervical estaban normalmente descompuestos, tanto como los que juntan las vértebras y las vértebras 

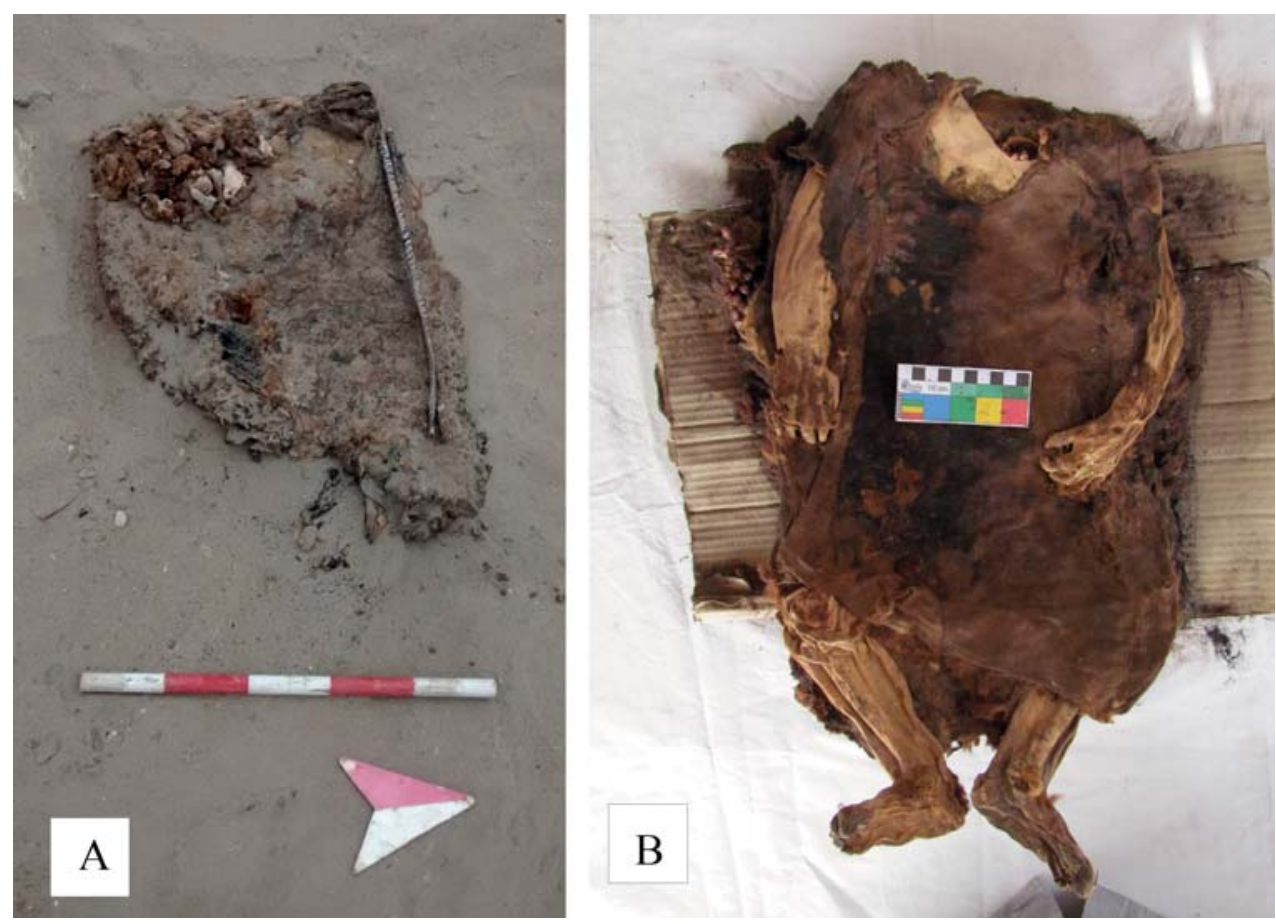

Fig. 4

con las costillas. De otro lado, se registró normalmente la conservación de los ligamentos de extremidades, en muchos casos también los de la pelvis (para contar solo los más importantes). Eso indica que los ligamentos que fijan las extremidades y el cráneo al cuerpo se descompusieron antes de momificarse, mientras los que se descomponen en un tiempo más prolongado si se momificaron. Lo que es importante para nosotros es, que - por la descomposición de los ligamentos más importantes - no es posible levantar el cuerpo momificado en tal manera sin romper su orden anatómico. Todos los cuerpos ubicados en los fardos conservados presentaban el orden anatómico completo, entonces podemos excluir la posibilidad que se sacaba los cuerpos de sus tumbas en tal manera como en la sierra. Cualquier acto de este tipo se terminaría con el desplazamiento de las extremidades y la cabeza, también las vértebras y las costillas, mientras en todos los casos nosotros ubicamos a esas partes in situ, sin ninguna evidencia de manipulación.

Un problema que hasta este momento no resolvemos, es la posibilidad de la extracción post-mortem de los cráneos. Como señalamos antes, esta tradición está bien estudiada para las culturas de la costa sur: Paracas y Nasca. Sin embargo, como indica la iconografía en los textiles de las culturas Ychma o Chimú, el uso de las cabezas momificadas se practicaba también en las sociedades tardías costeñas. En Cerro Colorado tuvimos algunos entierros donde faltaba el cráneo; sin embargo, eso 
se puede deber a la huaquería. Pero, en algunos casos, el cuerpo presentaba el orden anatómico, con la ausencia del cráneo, como en el caso de un individuo de edad de 6-12 meses, del CF 26, con su cuerpo momificado y el cráneo extraído un tiempo después de su muerte (Fig. 4). ¿Cómo y cuándo se los sacaba? No se ubicó ninguna evidencia que podrían sugerir que se cortó la cabeza cuando todavía estaba articulada. Entonces, probablemente, tenemos que ver con una extracción que ocurría semanas, meses o años después de la muerte. Lo interesante es que se sacaba cráneos tanto de los adultos como los niños, incluyendo recién nacidos. Además, eso nunca pasaba en los casos de fardos más elaborados, donde siempre ubicamos cuerpos enteros.

\section{Discusión}

Para sumar todo lo que presentamos hasta este momento, podemos concluir que el estudio estratigráfico y forense de los fardos y cuerpos excavados en los cementerios de la cultura Chancay permitió identificar episodios en que el fardo posiblemente fue excavado para añadir los siguientes textiles. Excluimos la interacción directa con el cuerpo, ya que no se le desenvolvía ni sacaba para que tomase parte en las ceremonias. Sin embargo, es posible que se sacaba los cráneos de algunos muertos un tiempo después de su muerte y enterramiento. Esas prácticas no eran comunes y se las identificó solo en el 1\% de más de 500 fardos funerarios estudiados.

Los resultados de nuestro estudio sugieren que en la cultura Chancay el culto a los ancestros si incluía el contacto directo con el fardo, pero sin interacción directa con el muerto. Podemos entender eso si recordamos, que la misma ceremonia del enfardelamiento fue tratada como una transformación del muerto al ancestro poderoso, como señalan Fung (1995) y Makowski (2005). En un estudio recién publicado, Arnold et al. (2016) informan de un ídolo ejecutado de oro, el dios Tata Quri de la comunidad moderna boliviana Qakachaca. Este dios se encuentra envuelto con textiles, para que tenga un estado semejante a la petrificación ("como si fuera una piedra"), pero también para controlar su poder. El enfardelamiento parece convertir el muerto a una entidad semejante a la piedra, entonces, al estado físico más perfecto en la cosmovisión andina (Salomon 1995). Como ya señalamos, en los Andes los ancestros son vistos también como semillas o "plantas tiernas". No sabemos si esas creencias compartían las sociedades de la cultura Chancay, pues a los muertos llamaban malqui o munao, el nombre que significaba "abuelo". La transformación del cuerpo a través del enfardelamiento podría explicar porque no se desenvolvían los muertos para interactuar con ellos: al convertirse en el fardo, el muerto constituía una sola entidad compuesta no solo por el cuerpo, sino por todos los materiales que lo envolvían.

Nuestro estudio señala también la extracción de cráneos de los fardos. El uso ritual de las cabezas humanas en el Periodo Intermedio Tardío está confirmado 
por la iconografía de las culturas contemporáneas a Chancay, como Chimú, Lambayeque e Ychma. Una de las cabezas falsas del cementerio de Macatón (valle de Chancay) analizada por nosotros contenía un fragmento del cráneo de un recién nacido, que en momento de la construcción de la cabeza ya estaba momificado, entonces este fragmento fue extraído de un contexto todavía más antiguo. Sin embargo, tenemos que incluir otras opciones, como la huaquería, pues en muchos casos se puede encontrar un rico ornamento metálico y textil colocado sobre el rostro, o envuelto alrededor, un fenómeno conocido por los huaqueros. La misma extracción con fines rituales pudo pasar también en el Periodo Colonial o aun en los tiempos modernos, ya que las calaveras constituyen un componente importante de la mesa del curandero. Recordemos que este fenómeno tocaba a los fardos modestos y poco complejos, mientras en los más elaborados siempre ubicamos cuerpos enteros. Llegamos a la misma conclusión que antes: en la cultura Chancay el culto a los ancestros no requería la interacción directa con el muerto, al menos cuando se trataba de los ancestros más importantes. De otro lado, la extracción de los cráneos solo en algunos casos puede indicar que existían ideologías diferentes en la misma sociedad, con modos diferentes de su expresión.

\section{Conclusión}

Durante el estudio estratigráfico de los fardos funerarios de la cultura Chancay ubicamos evidencias de la interacción póstuma en forma de la adición intencional de los textiles siguientes que podía pasar en dos modos: o por la envoltura o por la colocación en la cima del fardo. En el segundo caso los textiles colocados en la cima del fardo presentaban un estado de conservación mucho mejor que el fardo mismo, que sugiere que no estaban expuestos a los mismos factores tafonómicos que el fardo, siendo depositado un tiempo después. Además, observamos una capa de arena que separaba los fardos de los textiles externos, lo que se puede interpretar como la evidencia de que el fardo una vez enterrado fue excavado un tiempo después para añadir los textiles. A base de la observación de la articulación de los cuerpos se puede excluir el contacto directo con el muerto. Los cuerpos ubicados en los fardos siempre estaban articulados, lo que indica que no se los sacaba después de enfardelar. Sin embargo, en algunos casos tuvimos que ver con la extracción póstuma de los cráneos, un fenómeno que pudo pasar todavía en los tiempos prehispánicos, o ya después en los tiempos coloniales o aun modernos. La falta de interacción directa con el muerto puede indicar, que el cuerpo una vez envuelto y convertido en el fardo constituía una pieza solida con sus textiles y artefactos, y por eso no era necesario desenvolverlo, y el culto a los antepasados se realizaba por el contacto con el fardo, no el cuerpo directamente. La ausencia de cráneos registrada en algunos casos puede significar que en la cultura Chancay se practicaba el uso ritual de las cabezas de los antepasados en otras personas, 
percibidas como camasca (llenas por la cantidad extraordinaria de la energía vital, camac). Otra explicación posible es el saqueo de las tumbas tanto por curanderos coloniales y modernos que usan cráneos en sus mesas, como por huaqueros que buscan adornos elaborados, en muchos casos colocados cobre el rostro. Esperamos, entonces, que este articulo pueda ser una contribución en los estudios sobre el culto a los ancestros en el antiguo Perú.

\section{Agradecimientos}

Este proyecto se desarrolló bajo el auspicio de la Universidad Nacional Mayor de San Marcos de Lima, a través de los proyectos de investigación del Vicerrectorado de Investigación (131501115: "La Cultura Chancay en el Valle de Huaura"), cofinanciado por la Municipalidad Distrital de Santa María. La datación radiocarbónica y estudios arqueométricos fueron financiados por El Centro Nacional de Ciencia (NCN), ul. Twardowskiego 16, 30-312 Kraków, Polonia, como parte del proyecto "Biographies of the nameless (...)", numero de financiamiento UMO 2015/19/N/HS3/00552.

\section{Referencias citadas}

Altamirano Alfredo y José María Arguedas

2016 Wakanismo. El modelo del enfoque teórico andino. Arqueología y Sociedad 30:437 - 496.

Arnold, Denise, Juan de Dios Yapita y Elvira Espejo

2016 Wak'as, objetos poderosos y la personificación de la materialidad en los Andes meridionales: pugnas de exegesis sobre la economía religiosa según las experiencias del género. En Wak'as, diablos y muertos. Alteridades significantes en el mundo andino, editado por Lucilla Bugallo y Mario Vilca, pp. 31 - 71. Universidad Nacional de Jujuy - Instituto Francés de Estudios Andinos.

Arriaga, Pablo Joseph

1968 [1621] Extirpación de la idolatría del Piru. Biblioteca de Autores Españoles, tomo 209 (Crónicas Peruanas de Interés Indígena). Madrid: Ediciones Atlas.

Avila Francisco de

1987 [1598] Ritos y tradiciones de Huarochiri del siglo XVII (Dioses y Hombres de Huarochiri), editado por Gerard Taylor. Lima: Instituto de Estudios Peruanos/Instituto Frances de Estudios Andinos.

Bueno, Alberto

2012 Antecedentes arqueológicos del Precerámico y el Horizonte Medio en el valle de Huaura. Kullpi, investigaciones culturales en la provincia de Huaral y el Norte Chico 6:119-138. Lima. 
Buikstra, Jane

2019 Ortner's Identification of Pathological Conditions in Human Skeletal Remains. 3rd edition. Academic Press.

Buikstra, Jane y Dennis Ubelaker (eds.)

1994 Standards for Data Collection from Human Skeletal Remains. Field Museum of Natural History, Arkansas Archaeological Survey Research Series, $\mathrm{N}^{0} 44$.

Casas, Bartolomé de las

1892 [1550] De las antiguas gentes del Perú. Madrid, Tipografia de Manuel G. Hernández.

Chapman John y Bisseka Gaydarska

2006 Parts and Wholes: Fragmentation In Prehistoric Context. 264 pp. Oxford: Oxbow Books

Cieza de León, Pedro

1946 [1553] La crónica del Perú. 262 pp. México: Editorial Nueva España.

Cunningham Craig, Louise Scheuer. y Sue Black

2016. Developmental Juvenile Osteology. 2rd edition. Academic Press.

Diaz, Carla

2016 Cuerpo vegetal y violencia fecundadora en las fuentes coloniales andinas. Boletín del Museo Chileno de Arte Precolombino 21(2): 153

- 169. DOI: http://dx.doi.org/10.4067/S0718-68942016000200010

Duday, Henry

2009 The archaeology of dead. Lectures in Archaeothanatology. 230 pp. Oxbow Books, Oxford.

Eliade, Mircella

1974 Tratado de historia de las religiones. 133 pp. Tomo 2, Ediciones Cristianidad, Madrid.

Fitzsimmons, John

2009. Death and the Classic Maya Kings. 321 pp. University of Texas Press.

Frame, Mary, Daniel Guerrero Zevallos, Maria del Carmen Vega

Dulanto y Patricia Landa Cragg

2004 Un fardo funerario del Horizonte Tardio del sitio Rinconada Alta, valle de Rimac. Bulletin de Instituto francés de etudes andines 3(33): 815 - 860. DOI: DOI: 10.4000/bifea.5377

Fowler, Chris

2004 The archaeology of personhood. An anthropological approach. London: Routlege.

Fung, Rosa

1978

Análisis tecnológico de encajes del antiguo Perú: período tardío. En: Tecnología Andina, editado por Roger Ravines, pp. 333-345. Instituto de Estudios Peruanos. Lima. 
1995 Los encajes hechizados de la cultura Chancay. Boletín de la Fundación Museo Amano 7: 1-13. Lima.

Goring-Morris, Nigel

2002 The Quick and the Dead: The Social Context of Aceramic Neolithic Mortuary Practices as Seen from Kfar Ha Horesh. en Life in Neolithic Farming Communities Social Organization, Identity, and Differentiation, Holguín, Gonzalo editado por I. Kuijt, pp. 103 - 136. Kluvert Academic Publishers.

2007 [1608] Vocabulario de la Lengva General de todo el Perv llamada Lengva Qquichua o del Inca, Digitalizado por Runasimipi Qespisqa Software (http://www.runasimipi.org) para publicación en el internet, 2007.

Hollund, Hege Ingjerd, Malou Blank, Karl Göran - Sjögren

2018 Dead and buried? Variation in post-mortem histories revealed through histotaphonomic characterization of human bone from megalithic graves in Sweden. PLOS ONE 13(10): e0204662. DOI: https://doi. org/10.1371/journal.pone.0204662

Horkheimer, Hans

1970 Chancay prehispánico: diversidad y belleza. En: 100 años de arqueología en el Perú, editado por Roger Ravines, pp. 363-378. Petroperú. Lima.

Isbell, William $\mathrm{H}$.

1997 Mummies and mortuary monuments. A Postprocessual Prehistory of Central Andean Organization. 371 pp. Texas University Press.

Janway Robert C., Andrew C. Wilson, Gerardo Carpio - Díaz y Sonia Guillen

2009 Taphonomic Changes to the Buried Body in Arid Environments: An Experimental Case Study in Peru, en Criminal and Environmental Soil Forensics, editado por Karl Ritz, Lorna Dawson, David Miller, pp. 341 - 356. Springer Science + Business Media B.V. DOI: https://

Kaulicke, Peter doi.org/10.1007/978-1-4020-9204-6_22

1997 Contextos funerarios en Ancón. 123 pp. Pontificia Universidad Católica del Perú. Lima.

Krzanowski, Andrzej

1991 Estudios sobre la Cultura Chancay, Perú. (editor), Universidad Jaguellona.

2008 Kultura Chancay. Środkowe wybrzeże Peru. Polskie Towarzystwo Studiów Latynoamerykanistycznych, Instytut Amerykanistyki i Studiów Polonijnych UJ, 228 pp.

2016 Sitios amurallados o fortalezas Chancay en la Costa Central del Perú, en Ensayos sobre arqueología y etnología de los Andes peruanos. Wydawnictwo Uniwersytetu Jagiellońskiego. 
Majchrzak, Lukasz, Pieter van Dalen y Tomasz Goslar

En prensa Radiocarbon dating of the pre-Columbian Chancay funerary bundles. Radiocarbon.

Makowski, Krzysztof

2005 Deificación frente a ancestralización del gobernante en el Perú prehispánico: Sipán y Paracas, en Arqueología, geografía e historia: aportes peruanos en el 50to. Congreso de Americanistas, VarsoviaPolonia, 2000: 39 - 80. Fondo Editorial PUCP.

2010 Horizontes y cambios lingüísticos en la prehistoria de los Andes centrales. Boletín de Arqueología PUCP 10: 95 - 122.

Medina Martin Cecilia y Mira Vargas Sánchez

2007 Posthumous body treatments and ritual meaning in the Classic Period northern Petén. A taphonomic approach, en New Perspectives on Human Sacrifice and Ritual Body Treatments in Ancient Maya Society, editado por Vera Tiesler y Andrea Cucina, pp 102-119.. New York: Springer Science + Business Media, LLC. DOI: https://doi. org/10.1007/978-0-387-48871-4_5

Ortner, Donald

2003. Identification of Pathological Conditions in Human Skeletal Remains. Academic Press.

Pinheiro, Joao

2006 Decay Process of a Cadaver, en Forensic Anthropology and Medicine: Complementary Sciences From Recovery to Cause of Death, editado por A. Schmitt, E. Cunha, and J. Pinheiro, pp. 85 - 116. (C) Humana Press Inc., Totowa, NJ. DOI: https://doi.org/10.1007/978-1-59745-099-7_5

Plasencia Soto, Rommel

2015 Santiago, Illapa, la lluvia y el mundo de los ancestros en los Andes: a propósito de una re-lectura de Emio Choy. Alma Mater 2(3): 127 - 138.

Poma, Guaman

1980 [1615] Nueva crónica y buen gobierno. 2 tomos, 439 pp. Biblioteca Ayacucho, N. 75, Caracas.

Proulx, Donald

1999 Nasca Headhunting and the Ritual Use of Trophy Heads, en Nasca: Geheimnisvolle Zeichen im Alten Peru, editado por Judith Rickenbach, pp. 79-87. Zürich: Museum Rietberg Zürich, 1999.

Salas, José Antonio

$2008 \quad$ Acerca de la etimología de los munaos. B. APL. 46: 212 - 222.

Salomon, Frank

1995 The beautiful grandparents. En Tombs for the living. Andean mortuary practices, editado por Tom Dillehay, pp. 315 - 354. Washington D.C., Dumbarton Oaks. 
Szyjewski, Andrzej

2016 Etnologia religii. 635 pp. Zakład wydawniczy Nomos, Kraków,.

Takigami Mai, Shimada Izumi, Rafael Segura, Sarah Muno, Hiroyuki Matsuzaki, Fuyuki Tokanai, Kazuhiro Kato, Hitoshi Mukai, Omori Takayuki y Minoru Yoneda

2014 Assesing the chronology and rewrapping of funerary bundles at the prehispanic religious center of Pacahcamac, Peru. Latin American Antiquity 25(3): 322 - 343. DOI: https://doi.org/10.7183/10456635.25.3.322

Taylor Gerard

2000 Camac, camay y camasca en el manuscrito quechua de Huarochirí, en Camac, camay y camasca y otros ensayos sobre Huarochirí y Yauyos, pp. 1 - 17 Lima, Perú: Institut français d'études andines; Cuzco: Centro Tello, Julio Cesar de Estudios Regionales Andinos "Bartolomé de Las Casas".

2015 Arqueología del territorio Chancay. En: Cuadernos de Investigación del Archivo Tello 11. 183 pp. Editado por Museo de Arqueología y Antropología de la Universidad Nacional Mayor de San Marcos. Lima.

Van Dalen Luna, Pieter

2008 Los ecosistemas arqueológicos en la cuenca baja del río Chancay Huaral. Su importancia para el desarrollo de las formaciones sociales prehispánicas. Ed. Gutemberg. Lima.

2012 Arqueología tardía del valle Chancay-Huaral: Identificando la nación Chancay. Investigaciones sociales, 28: 271-284. Instituto de Investigaciones Sociales, Facultad de Ciencias Sociales. Universidad Nacional Mayor de San Marcos. Lima. DOI: https://doi.org/10.15381/ is.v16i28.7403

2016 Estrategias de dominación Tawantinsuyu en el complejo arqueológico de Lumbra, valle medio del río Chancay, provincia de Huaral. Tesis para optar el grado de Magíster en Arqueología Andina. Unidad de Post Grado, Facultad de Ciencias Sociales, Universidad nacional mayor de San Marcos. Lima.

2017 Sacachispa: un cementerio de agricultores Chancay en Huando, Huaral. Ed. Gutemberg. Lima.

2019 Vivir en la cima del mundo: identificación de las secuencias ocupacionales en Marca Piche, un sitio Atavillos en San José de Baños, Huaral: Temporada 2017. Actas del V Congreso Nacional de Arqueología: 267-280. Volumen I. Ministerio de Cultura. 2019. Lima. 
Van Dalen Luna, Pieter y Lukasz Majchrzak

2019 The stratigraphy of the pre-Columbian Chancay funerary bundle. Open Journal for Studies in History 2(1): 1 - 18. DOI: https://doi. org/10.32591/coas.ojsh.0201.01001v

en prensa Identificación y cronología de las costumbres funerarias en los fardos de la cultura Chancay de Cerro Colorado.

Van Dalen Luna, Pieter, Łukasz Majchrzak, Kamilla Malek, Joanna Kuncewicz, Paweł Miśkowiec

2020 The multimodal chemical study of the pre-Columbian Peruvian mummies. Analyst 145: 5670 - 5681. DOI: https://doi.org/10.1039/ D0AN01017K

Van Dalen Luna, Pieter, Alfredo Altamirano y Łukasz Majchrzak

2018 Marcas para la vida, señales para la muerte. Los cuerpos tatuados de la cultura Chancay en Cerro Colorado, Huacho, Perú. Revista M. Estudos sobre a morte, os mortos o morrer 3(6):344 - 377. DOI: http://dx.doi. org/10.9789/2525-3050.2018.v3i6.344-377 
Tabla 1. Descripción sumaria de los contextos funerarios discutidos en el artículo.

\begin{tabular}{|c|c|c|c|c|c|c|}
\hline 岂 & 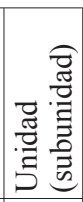 & 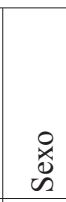 & $\begin{array}{l}\tilde{\pi} \\
\text { I } \\
\end{array}$ & 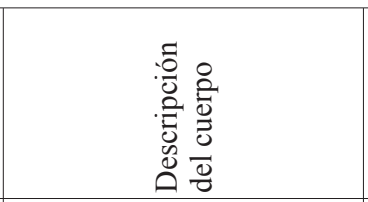 & 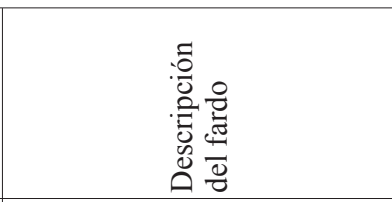 & 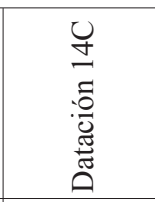 \\
\hline $\begin{array}{l}\text { "Gran } \\
\text { Fardo" }\end{array}$ & 30 & $\mathrm{M}$ & $\begin{array}{l}50- \\
70\end{array}$ & $\begin{array}{l}\text { Cuerpo completo, en la } \\
\text { posición flexionada, las } \\
\text { manos sobre el rostro. Mo- } \\
\text { mificación parcial de la piel } \\
\text { de las extremidades y las } \\
\text { manos. Cabello conservado } \\
\text { Osteoartritis en las articula- } \\
\text { ciones (la más desarrollada } \\
\text { en las articulaciones de } \\
\text { codos), las vértebras del } \\
\text { tramo lumbar con osteofi- } \\
\text { tos. Desplazamiento de la } \\
\text { cabeza del fémur derecho. } \\
\text { El cuerpo mantiene orden } \\
\text { anatómico. } \\
\end{array}$ & $\begin{array}{l}\text { El individuo con una lámina } \\
\text { de cobra en forma de me- } \\
\text { dialuna en el frente, sobre } \\
\text { la cabeza se encuentra una } \\
\text { redecilla, alrededor corre una } \\
\text { honda ceremonial. El cuerpo } \\
\text { cubierto por una capa de } \\
\text { algodón, luego envuelto con } \\
4 \text { telas en mal estado de con- } \\
\text { servación - conforman una } \\
\text { compactación. Sobre el textil } \\
\text { exterior (parte superior del } \\
\text { fardo) se coloca una capa de } \\
\text { arena de espesor de } 4 \text { cm, y } \\
\text { luego se encuentran } 37 \text { texti- } \\
\text { les (1 tela con decoración por } \\
\text { brocado) colocados sobre la } \\
\text { parte anterior del fardo. }\end{array}$ & $\begin{array}{l}654 \pm 30 \mathrm{BP} \\
1300- \\
1401 \text { d.C. }\end{array}$ \\
\hline 20 & 2 & $F$ & $\begin{array}{l}30- \\
40\end{array}$ & $\begin{array}{l}\text { Cuerpo completo, en la } \\
\text { posición flexionada, las } \\
\text { manos sobre el rostro. Mo- } \\
\text { mificación parcial de la piel } \\
\text { de las extremidades, cabe- } \\
\text { llo conservado. Osteoar- } \\
\text { tritis en las articulaciones, } \\
\text { las vértebras lumbares con } \\
\text { osteofitos. Deformación } \\
\text { cefálica tabular erecta. La } \\
\text { boca rellenada con algo- } \\
\text { dón mezclado con láminas } \\
\text { pequeñas de cobre. Dedos } \\
\text { atados con fibra de maguey. } \\
\text { Tiene tatuaje en forma de } \\
\text { hileras de puntos (antebra- } \\
\text { zo izquierdo, cara dorsal). } \\
\text { El cuerpo mantiene orden } \\
\text { anatómico. }\end{array}$ & $\begin{array}{l}\text { Cuerpo envuelto con una } \\
\text { capa de algodón, luego con } \\
5 \text { mantas llanas de algodón } \\
\text { y fragmentos de piel animal, } \\
\text { posiblemente de perro. Sobre } \\
\text { la cima de última manta (lado } \\
\text { anterior del fardo) se coloca } \\
\text { una capa de arena, de espesor } \\
\text { de } 4 \mathrm{~cm} \text {. Sobre esa capa se } \\
\text { coloca un textil llano de algo- } \\
\text { dón de color anaranjado, con } \\
4 \text { láminas de cobre cosidas. } \\
\text { Luego se registran tres paños } \\
\text { decorados, colocados sobre } \\
\text { el lado anterior del fardo, y } \\
\text { finalmente dos mantos que } \\
\text { envuelven todo el paquete. Al } \\
\text { fardo se sujeta una red. }\end{array}$ & $\begin{array}{l}800 \pm 30 \\
\text { BP 1220- } \\
1290 \text { d.C. }\end{array}$ \\
\hline 26 & \begin{tabular}{|l}
10 \\
$(12)$
\end{tabular} & $F$ & $\begin{array}{l}6- \\
12 \\
\text { me- } \\
\text { ses }\end{array}$ & $\begin{array}{l}\text { Cuerpo sin cabeza la cual } \\
\text { fue cortada (junto con el } \\
\text { cuello) con una herramien- } \\
\text { ta aguda (posiblemente } \\
\text { un cuchillo), postmortem. } \\
\text { Todo el cuerpo momifica- } \\
\text { do, excepto para su espal- } \\
\text { da. Dedos atados con fibra } \\
\text { de maguey. No se registran } \\
\text { patologías. El cuerpo man- } \\
\text { tiene orden anatómico. }\end{array}$ & $\begin{array}{l}\text { Cuerpo cubierto con una capa } \\
\text { fina del algodón desmontado } \\
\text { vestido en una túnica sin man- } \\
\text { gas. Luego fue envuelto con } \\
\text { cuatro textiles más, entre los } \\
\text { cuales se colocaba algodón } \\
\text { fino. }\end{array}$ & \\
\hline
\end{tabular}

